\title{
Medical Image Retrieval System using 2D-DWT and Convolutional Neural Network
}

\author{
B. Nandhini, K. Ribana and S. Pradeep \\ Department of ECE, M. Kumarasamy College of Engineering, Karur - 639113, Tamil Nadu, India; \\ nandhinib.ece@mkce.ac.in, ribanak.ece@mkce.ac.in, sspradeep20@gmail.com
}

\begin{abstract}
Objectives: To propose an algorithm for Medical image retrieval better. Methods/Statistical analysis: The proposed image retrieval system incorporates with methods like discrete wavelet transform, information gain and the convolutional neural network for classification. Findings: We propose an algorithm of image retrieval that implements the feature extraction with 2D-Discrete Wavelet Transform (DWT), information gain for feature reduction and convolutional neural network for classification. The feature extraction involves the extraction of features from medical image using DWT. The Feature reduction was carried out by information gain. The image is classified by using classifiers such as convolutional neural network, traditional SVM classifier and KNN classifier. Application/Improvements: The classifier result provides the similar images in the database. The classification accuracy of convolutional neural network is $91.25 \%$ outperforms the traditional SVM classifier and KNN classifier. The image retrieved on high accuracy classifier provides exact match of query medical image for the diagnosis and treatment schedule.
\end{abstract}

Keywords: Convolutional Neural Network, Discrete Wavelet Transform, Information Gain, KNN Classifier, Medical Image Retrieval

\section{Introduction}

Medical database provides the disease stage and treatment summary of individual patient with medical images. Image retrieval of particular disease summary provides the analysis of various stages of disease and diagnosis results. 1 The challenge of medical image retrieval from huge database is handled by various approaches. Initially, textual annotations of images have been used for retrieval. The keyword search of image is the process incorporated in textual based retrieval system. In this process, the major drawback is of labor work and time consumption.., 3 The next stage of image retrieval system uses query based image search known as semantic queries. The efficient method of image retrieval using the content of image rather than the textual annotation and semantic queries provides the accurate results and information for diagnosis.
The efficient information from the medical image is used for the diagnosis plan and treatment schedule for the patients. The most commonly used imaging techniques for accurate information for diagnosis are CT, PET, MRI, $\mathrm{X}$-Rays. The annotated keywords of the image are stored as a traditional image database. The text-annotated image database is time consuming, expensive and laborious. The drawback of textual annotation cannot embed with all the information in the medical image and it is difficult to represent all the information the form of keywords. The low level features such as texture, color and shape involves in content based image retrieval system. This replaces traditional method of image retrieval system. The image retrieval system retrieves the image with mutual correspondence between input query image and images in the database.

The image retrieval system uses and features extracted from an image and compare the features in the database to

*Author for correspondence 
retrieve similar image. Features can be color, texture, and shape for content base image retrieval. The most familiar feature extraction methods can be DST, DCT and DWT. The features of all images in the database was extracted and stored as a feature database. The images are classified using machine learning algorithms such as decision tree, KNN, K-means clustering, linear regression, SVM, neural network and fuzzy classifier. The feature matching done by using image distance measure. The process of Medical image retrieval system is shown in Figure 1.

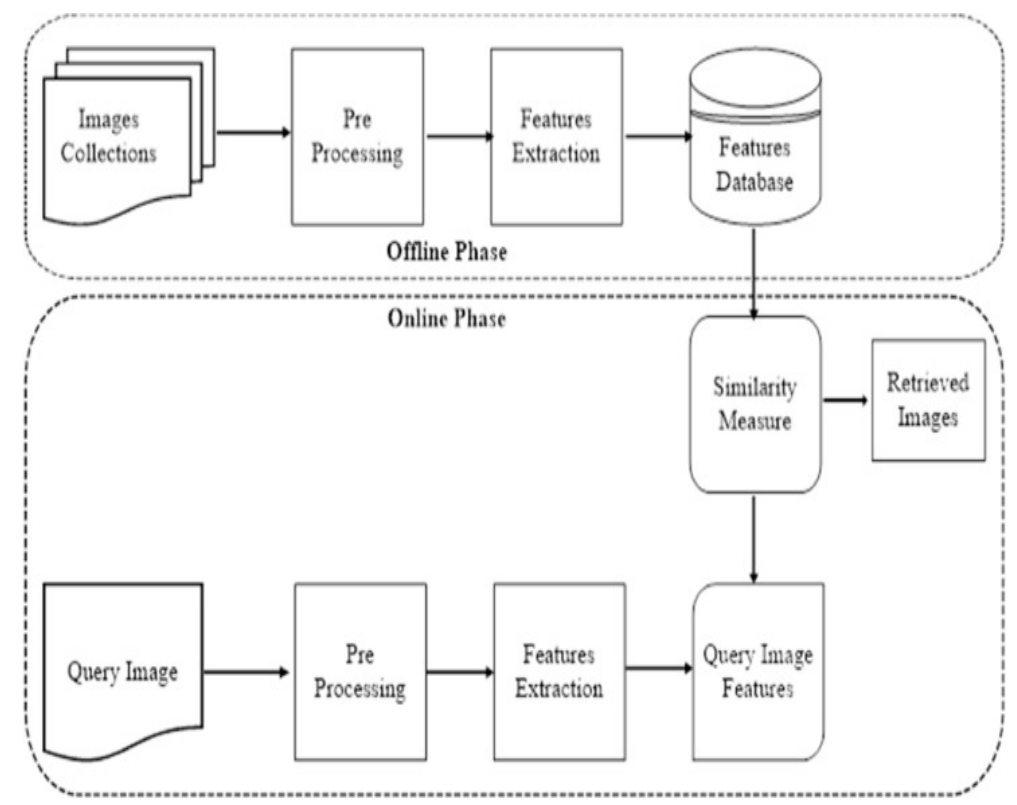

Figure 1. Image retrieval system.

\section{Previous Research}

The feature extraction process was done by Discrete Sine transform. ${ }^{4}$ The Gaussian kernel based classification used for the training and testing to retrieve image from the database.

A technique of high level feature extraction technique for efficient medical image retrieval. $\frac{5}{-}$ This paper proposes a feature extraction by discrete wavelet transform for various level of feature extracted from single image.

The algorithm for image retrieval using radon transform and convolutional neural network was explained. ${ }^{6}$ In this paper, $\mathrm{CNN}$ generates the convolutional kernel automatically which improves the classification accuracy and timing behavior of image retrieval system.

\section{Research Methods}

This section explores the concepts of 2-D Discrete Wavelet Transform, Information gain, KNN classifier, SVM classifier, Convolution neural network classifier for the proposed work

\subsection{Image Database}

The Database is a collection of medical images with 5 classes provided by the Aachen university if technology. Among those images $70 \%$ of image were used for training phase and $30 \%$ of images used for testing phase.

\subsection{Feature Extraction}

The feature extraction process is achieved by $2 \mathrm{D}-\mathrm{DWT}$. The Images represents the multidimensional signals. The decomposition of images extends to multidimensional wavelets. The discrete wavelet transform converts an image to various frequency bands with the multiresolution property.

The Discrete wavelet transforms process with decomposition of CT or MRI images into sub-bands at various frequency levels. The first and second stage of decomposition was shown in Figure 2. The various stage of decomposition can be undergone with multistage application of 2D-DWT. The LL band in every decomposition gives approximate information of a medical image. The LH, HL, HH represents the vertical, horizontal and diagonal sub bands. These bands incorporated with high level information of images such as edges and sharpness. 


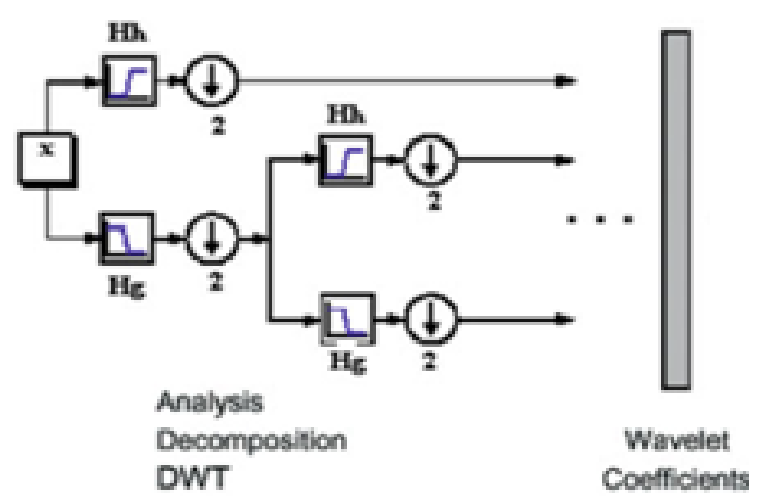

Figure 2. Discrete wavelet transform.

The 2D-DWT operation can be done using 2D filtering and down sampling. Let $Y_{x, z}$ be an image with two dimension. The image will be transformed through the two stages of analysis filters $\mathrm{h}$ and $\mathrm{g}$ and sampled by two. Analogous to the $1 \mathrm{D}-\mathrm{DWT}$, the $2 \mathrm{D}-\mathrm{DWT}$ is decomposed into a sum of fine to coarse resolution smooth coefficients. The coefficients for the first scale are given by the following formulas:

$$
\begin{aligned}
& \mathrm{W}_{\mathrm{z}, \mathrm{x}, \mathrm{l}}^{(\mathrm{d})}=\sum_{\mathrm{K}=0}^{\mathrm{L}-1} \sum_{\mathrm{l}=0}^{\mathrm{L}-1} \mathrm{~h}^{0} \mathrm{Y}_{2 \mathrm{z}+1-\mathrm{k} \bmod \mathrm{M}, 2 \mathrm{x}+1-\mathrm{lmod} \mathrm{N}} \\
& \mathrm{W}_{\mathrm{z}, \mathrm{x}, \mathrm{l}}^{(\mathrm{v})}=\sum_{\mathrm{K}=0}^{\mathrm{L}-1} \sum_{\mathrm{l}=0}^{\mathrm{L}-1} \mathrm{~h}^{1} \mathrm{Y}_{2 \mathrm{z}+1-\mathrm{k} \bmod \mathrm{M}, 2 \mathrm{x}+1-\operatorname{lmod} \mathrm{N}} \\
& \mathrm{W}_{\mathrm{z}, \mathrm{x}, \mathrm{l}}^{(\mathrm{h})}=\sum_{\mathrm{K}=0}^{\mathrm{L}-1} \sum_{\mathrm{l}=0}^{\mathrm{L}-1} \mathrm{~h}^{2} \mathrm{Y}_{2 \mathrm{z}+1-\mathrm{kmod} \mathrm{M}, 2 \mathrm{x}+1-\operatorname{lmod} \mathrm{N}} \\
& \mathrm{V}_{\mathrm{z}, \mathrm{x}, \mathrm{l}}=\sum_{\mathrm{K}=0}^{\mathrm{L}-1} \sum_{\mathrm{l}=0}^{\mathrm{L}-1} \mathrm{~h}^{3} \mathrm{Y}_{2 \mathrm{z}+1-\mathrm{k} \bmod \mathrm{M}, 2 \mathrm{x}+1-\mathrm{l} \bmod \mathrm{N}}
\end{aligned}
$$

Where the filters $\mathrm{h} 0, \mathrm{~h} 1, \mathrm{~h} 2$ and $\mathrm{h} 3$ represents the products component of the quadrature mirror filters $h$ and $g$.

$$
\begin{aligned}
& \text { h0 }=\text { h.h } h^{\mathrm{T}}, \mathrm{h} 1=\mathrm{h} \cdot \mathrm{g}^{\mathrm{T}} \\
& \mathrm{h} 2=\mathrm{g} \cdot \mathrm{h}^{\mathrm{T}}, \mathrm{h} 3=\mathrm{g} \cdot \mathrm{g}^{\mathrm{T}}
\end{aligned}
$$

Where $\mathrm{W}_{\mathrm{z}, \mathrm{x}, \mathrm{l}}^{(\mathrm{d})}, \mathrm{W}_{\mathrm{z}, \mathrm{x}, \mathrm{l}}^{(\mathrm{v})}, \mathrm{W}_{\mathrm{z}, \mathrm{x}, \mathrm{l}}^{(\mathrm{h})}, \mathrm{V}_{\mathrm{z}, \mathrm{x}, \mathrm{l}}$ are the coefficients from the diagonal, vertical, horizontal and scaling. The image $x(s, t)$ can be decomposed by using wavelet equations. The feature will be extracted using line measure technique.

\subsection{Information Gain}

The feature reduction process carried out in extracted feature to facilitate efficient image classification. The traits in the feature database can be reduced using Information gain calculation. In feature reduction process, the redundant information and noisy features removed from the database. This led to an efficient and optimize machine learning algorithm. The features are ranked and classified based on the values of information gain. This process extracts the high level features for classification and image retrieval process.

Information gain $\left(\mathrm{I}_{\mathrm{G}}\right)$ analyse the significant qualities of features with the values associated in the class of information gain measurement. The entropy is denoted as " $\mathrm{H}$ ".

$$
\mathrm{I}_{\mathrm{G}}(\mathrm{C}, \mathrm{A})=\mathrm{H}(\mathrm{C})-\mathrm{H}(\mathrm{C} \mid \mathrm{A})
$$

$\mathrm{C}$ represents class, a represents attribute, $\mathrm{I}_{\mathrm{G}}$ represents information gain.

Information gain algorithm ranked the features and distributes the missing values and numerical binary values in terms of frequency. The supports binary and nominal class.

\subsection{Classification}

The machine learning classifier such as K-Nearest Neighbor classifier, SVM classifier and Convolutional neural network can be used for classification.

\subsubsection{KNN Classifer}

The machine learning algorithm categorized into supervised learning and unsupervised learning. The KNN algorithm classified in supervised learning technique. The algorithm works on simple classification. It retrieves the image with distance measure between the test image and trained images in database. The KNN algorithm takes the test sample from input query image and finds the K-nearest neighbor in the trained database. The database assigns class to all the samples in the database. The class can be predicted by assigning test image to a majority classes and the nearest neighbor of the test image can be identified by the features in the distance measure. Let $\mathrm{T}$ be the training set defined by

$$
\mathrm{T}=\left\{\left(\mathrm{X}_{\mathrm{i}}, \mathrm{C}_{\mathrm{j}}\right) ; \mathrm{i}=1, \ldots, \mathrm{N}\right\}
$$

Where $C_{j}$ belongs to $C, J=1, \ldots J$ represents membership to a class and each features from the data is a vector $\mathrm{X}_{\mathrm{i}}=\left\{\mathrm{X}_{\mathrm{i} 1}, \mathrm{X}_{\mathrm{i} 2}, \mathrm{X}_{\mathrm{i} 3} \ldots ., \mathrm{X}_{\mathrm{ip}}\right\}$ defined by $\mathrm{p}$ descriptive attributes. This algorithm measures the distance $\mathrm{d}$ between the test image features and all the other features in the training database and then determines the K-nearest neighbors feature vector $\mathrm{X}$. The nearest neighbor feature is similar feature of the image to be retrieved. 


\subsubsection{SVM Classifier}

Support Vector Machines (SVMs) is the Machine learning process initiated from the statistical learning theory. It addresses the classification and regression problems. Its training algorithm is different from other that it allocates examples to each category in the learning process. The SVM aims to a construct model that depends on the

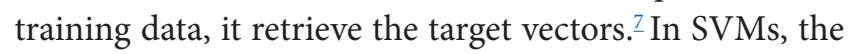
kernel function is used for non linear mapping. The non linear mapping maps the input data to a target vector even in high dimensional feature space. The hyper plane is generated to match the feature space within the training and testing phase. The SVM kernel functions can be in the form of, sigmoid functions. $\frac{899}{9}$ The support vectors are the term used in the training data phase. The training and testing will do separately by quadratic program with software programs.

\section{Convolutional Neural Network}

A neural network is a machine that is designed to model the way in which the brain performs a particular task or function of interest. The neural network is made up of artificial neurons. The neural network is usually implemented by using electronic components or is simulated in software on a digital computer.

A feed forward artificial neural network is termed as convolutional neural network specially designed to use minimum image preprocessing. The Convolutional neural network works on raw data. The classifier has two stage training and testing. During Training stage, CNN extract the features from the database and categorize into sub classes. $\frac{10,11}{1}$ The $\mathrm{CNN}$ process is shown in Figure 3.

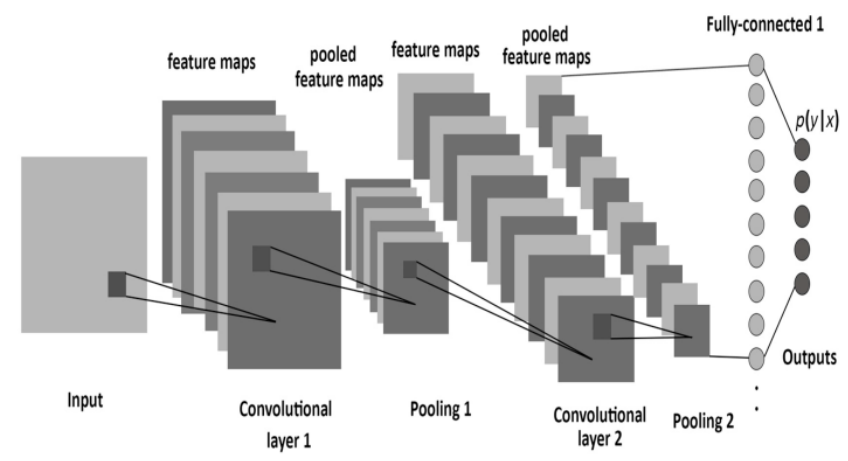

Figure 3. Convolution neural network process.
In $\mathrm{CNN}$, the convolutional kernals were generated by neural network which is major part for feature extraction. Thus CNN shows a better performance than all other classifiers in image classification.

\section{Results and Discussion}

The Database of 70 medical images were used for experimental setup which contains five classes of labels. The algorithm was implemented in MATLAB R2014b. The query image was given as shown in Figure 4. The result obtained from convolutional neural network is shown in Figure 5. The classification accuracy of proposed algorithm has validated higher than KNN and SVM classifier and shown in Figure 6.

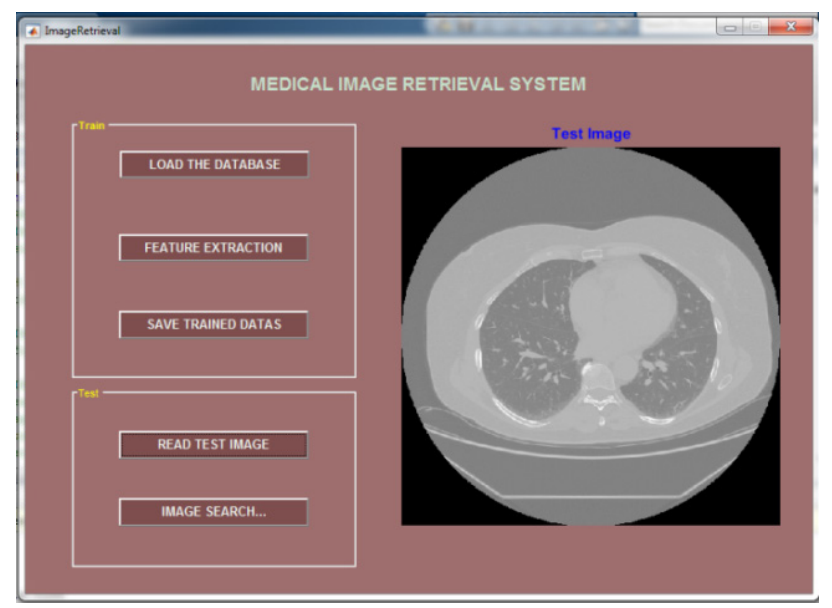

Figure 4. Query image.

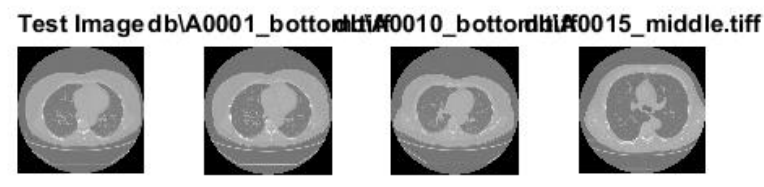

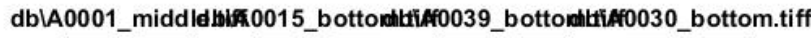
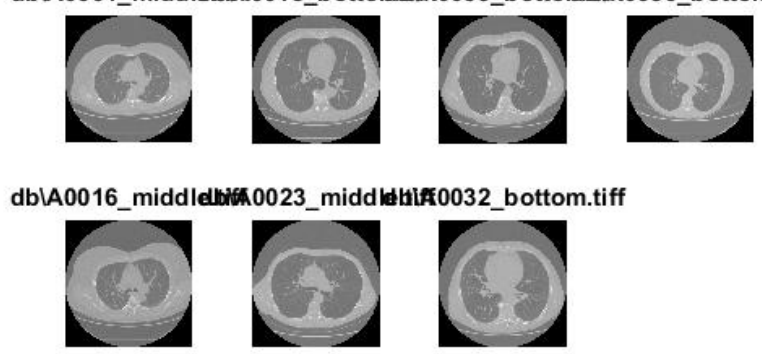

Figure 5. CNN image retrieval output. 


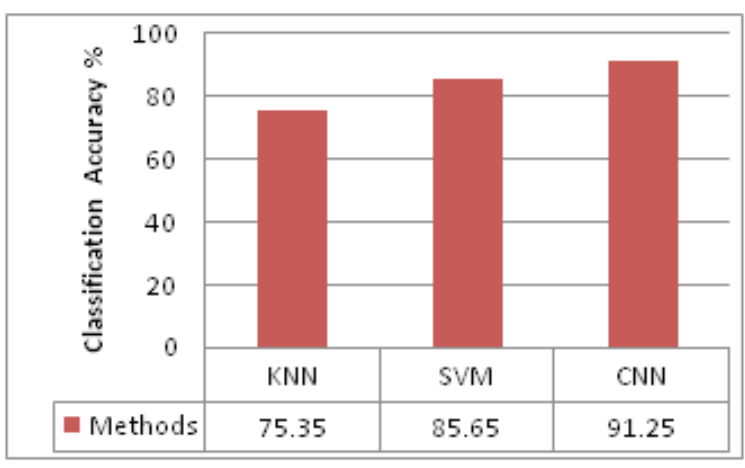

Figure 6. Classification accuracy.

\section{Conclusion}

In this paper, a novel approach for Medical image retrieval System is proposed with feature extraction and image classification. The query image was given through GUI of MATLAB. The features extracted using 2D-DWT and feature reduction process done by Information gain. The convolutional neural network is used for classification and by using distance measure the image retrieved from the database. The proposed algorithm improves the classification accuracy of about $5 \%$ than existing algorithms.

\section{References}

1. Müller H. A review of content-based image retrieval systems in medical applications clinical benefits and future directions. International journal of medical informatics. 2004; 73(1):1-23.
2. Mei-Ling S, Shu-Ching C, Chen M, Zhang C, Sarinnapakorn $\mathrm{K}$. Image database retrieval utilizing annuity relationships. Molecular Modeling Database; 2003. p. 78-85.

3. Barbu T. Content-based image retrieval using Gabor filtering. $20^{\text {th }}$ International Workshop on Database and Expert Systems Application; 2009. p. 236-40.

4. Balafar MA. Review of brain MRI image segmentation methods. Artificial Intelligence Review. 2010; 33(3): 261-74.

5. Koller TM. Multiscale detection of curvilinear structures in 2-D and 3-D image data. Proceedings of IEEE International Conference on Computer Vision; 1995. p. 864-9.

6. Lei $\mathrm{W}$, Jin R, Jain AK. Tag completion for image retrieval. IEEE Transactions on Pattern Analysis and Machine Intelligence. 2013; 35(3):716-27.

7. Dunn D, Higgins WE. Optimal Gabor filters for texture segmentation. IEEE Transactions on Image Processing. 1995; 4(7):947-64.

8. Chih-Chin L, Cheng-Chih T. Digital image watermarking using discrete wavelet transform and singular value decomposition. IEEE Transactions on instrumentation and measurement. 2010; 59(11):3060-3.

9. Feng-Lin C. System and method for information retrieval with barcode using digital image capture devices. U.S. Patent Application No. 11/350,030. 2006.

10. Hiremath PS, Pujari J. Content based image retrieval using color, texture and shape features. $15^{\text {th }}$ International Conference on Advanced Computing and Communications (ADCOM 2007); 2007. p. 780-4.

11. Lei W, Jin R, Jain KA. Tag completion for image retrieval. IEEE Transactions on Pattern Analysis and Machine Intelligence. 2013; 35(3):716-27. 